\title{
MAGNETIC FIELDS IN AM HER BINARIES
}

\author{
KINWAH WU ${ }^{1}$, PAUL A. MASON ${ }^{2}$ \\ 1.RCfTA, The University of Sydney, NSW 2006, Australia \\ 2. Department of Astronomy, Case Western Reserve \\ University, Cleveland, $\mathrm{OH} 44106$, USA
}

\section{Introduction}

There is growing evidence of complex white dwarf magnetic fields in CVs (e.g. Meggitt \& Wickramasinghe 1989; Robinson \& Córdova 1994). The properties of systems with non-dipolar white dwarf magnetic fields have been investigated, and it was found that a non-negligible quadrupole component is essential for the magnetic locking of AM Her binaries (AM Hers) (Wu \& Wickramasinghe 1993). In a recent study the ratio of the quadrupole to dipole component of the surface field of white dwarfs was shown to grow by a factor of 10 during the white dwarf cooling lifetime (Muslimov, van Horn \& Wood 1995), thus the quadrupole field component is long lived and significant.

\section{Synchronous versus asynchronous systems}

For synchronous AM Hers, it is generally not easy to distinguish between an offset dipole field and a dipole-quadrupole field. Nevertheless if a system has more than two accretion poles the evidence for a multipole field is strong. Systems with a multipole field also have other observational properties that distinguish them from the systems with a pure dipole field. If a system has a dipole field, the polar field strengths deduced from the cyclotron spectra are about a factor of 1.5 higher than the photospheric magnetic fields deduced from the Zeeman lines. This, however, does not hold for dipole-quadrupole fields. In fact, the suggestion that BL Hyi has a strong quadrupole component for its white dwarf magnetic field is based on this argument (Schwope 1995).

For asynchronous AM Hers, the accretion stream impacts different parts of the white dwarf magnetosphere throughout the spin-orbit beat cycle. For a dipole-quadrupole field (Fig. 1), the two main accretion regions are located near the intersection of the magnetic equator and the orbital plane,

A. Evans and J. H. Wood (eds.), Cataclysmic Variables and Related Objects, 203-204.

(c) 1996 Kluwer Academic Publishers. Printed in the Netherlands. 


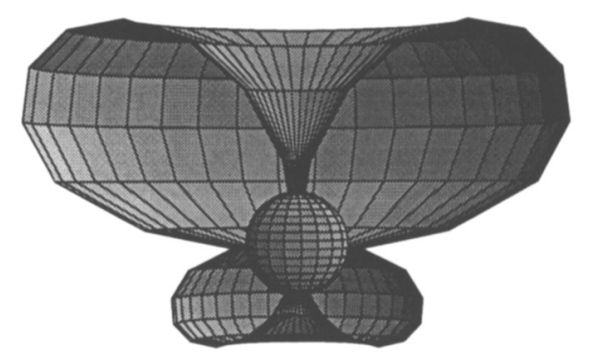

Figure 1. A white dwarf with comparable quadrupole and dipole field strengths

and accretion switches back and forth between the two poles with the spinorbit beat period. The field strength of the two poles are generally similar and have the same polarity, thus the polarization of the cyclotron emission they emit have same sign. At certain beat phases accretion onto a third (or even a fourth) accretion pole may also occur.

BY Cam is a typical example of an asynchronous AM Her system (e.g. Ramsay \& Mason 1996). In the model proposed by Mason et al. (1995) the main two accretion regions appear to be diametrically opposed but show the same sign of polarization (see Mason, Liebert \& Schmidt 1989). As a note of caution we point out that a competing model for BY Cam exists, in which the dominant spike in the periodogram of Ramsay \& Mason's figure 1 is the spin period rather than a side-band period and the spinorbit beat period is $7.07 \mathrm{~d}$ rather than $14.15 \mathrm{~d}$. However, the periods shown in Ramsay \& Mason's figure 1 are not readily explained in terms of the $7.07 \mathrm{~d}$ beat period model. In any case there is strong evidence for a complex field for the white dwarf in BY Cam due to extremely complex and variable polarization curves.

\section{Conclusions}

AM Her binaries generally have complex white dwarf magnetic fields. The field structure can be easier determined if the systems are asynchronous. Observations indicate that the magnetic field of BY Cam likely has a strong quadrupole component.

\section{References}

Mason, P.A., Andronov, I.L., Kolesnikov, S.V., et al., 1995, in "Magnetic Cataclysmic Variables", eds D. Buckley, B. Warner, ASP Conf. Ser. 85, 493

Mason, P.A., Liebert, J., Schmidt, G.D., 1989, Ap. J., 346, 941

Meggitt, S.M.A., Wickramasinghe, D.T., 1989, MNRAS, 236, 31

Muslimov, A.G., van Horn, H.M., Wood, M.A., 1995, Ap. J., 442, 758

Ramsay, G., Mason, P.A., 1996, these proceedings, p213

Robinson, C.R., Córdova, F.A., 1994, Ap. J., 437, 436

Schwope, A., 1995, Rev. Mod. Astron., in press

Wu K., Wickramasinghe D.T., 1993, MNRAS, 260, 141 